\title{
A more accurate tool for documenting functional endoscopic sinus surgery
}

\author{
Sean Fang ${ }^{1}$, Humera Babar-Craig ${ }^{1}$, and Natasha Choudhury ${ }^{1}$ \\ ${ }^{1}$ East Surrey Hospital
}

October 12, 2020

\begin{abstract}
Background: FESS encompasses numerous component procedures including antrostomy, ethmoidectomy, sphenoidotomy and frontal sinusotomy. The extent of FESS procedure will vary between patients depending on indications, imaging and surgical experience. Each procedure is remunerated according to procedural components with each component assigned an OPSC-4 cost code. In NHS hospitals, this relies on clinical coders' interpretation from the operation note, and may potentially be subject to incorrect coding and remuneration. Background: We have devised a FESS documentation tool which includes a tick box system for each component and allied procedures performed, together with relevant OPSC-4 codes. The aim of this novel FESS operation note is to improve accuracy of coding for procedures, avoid misinterpretations and ensure accurate remuneration for FESS procedures. Design: Retrospective quality improvement study. Methods: The tool was implemented in our hospital in April 2019. Codes and costs applied to each patient undergoing FESS for a 6-month period between April to October 2019 were compared for all cases performed during the same 6 month period in 2018. Data review of coding information was performed between the two time periods including T-test analysis to calculate for statistical significance. Results: 66 patients underwent FESS in 2018 compared to 70 patients in 2019, during the equivalent 6 month period. The tool was not used in two cases in the 2019 cohort. In 2018, the average cost applied to each FESS case was $£ 1,676$, compared to $£ 1,953$ per patient in the 6 months after the tool was implemented. This resulted in an average uplift of $£ 277$ in revenue per patient where the tool was used ( $\mathrm{p}=0.003$ ), due to more accurate capture and coding of FESS component parts. On average ,approximately 140 patients undergo FESS per year in our department; using the newly devised FESS documentation tool, we can estimate a potential increase in revenue of $£ 38,780$ per annum for FESS procedures alone. Conclusion: Our novel documentation tool has improved the clarity of recording endoscopic sinus surgery allowing more accurate interpretation and application of OPSC-4 coding. It also aids in understanding complex composite procedures and can be extended to other surgical specialties.
\end{abstract}

\section{Hosted file}

FESS tool 1.pdf available at https://authorea.com/users/366513/articles/486351-a-moreaccurate-tool-for-documenting-functional-endoscopic-sinus-surgery 


\section{NHS}

Surrey and Sussex Healthcare

NHS Trust

Ear nose and throat (ENT) FESS operation record

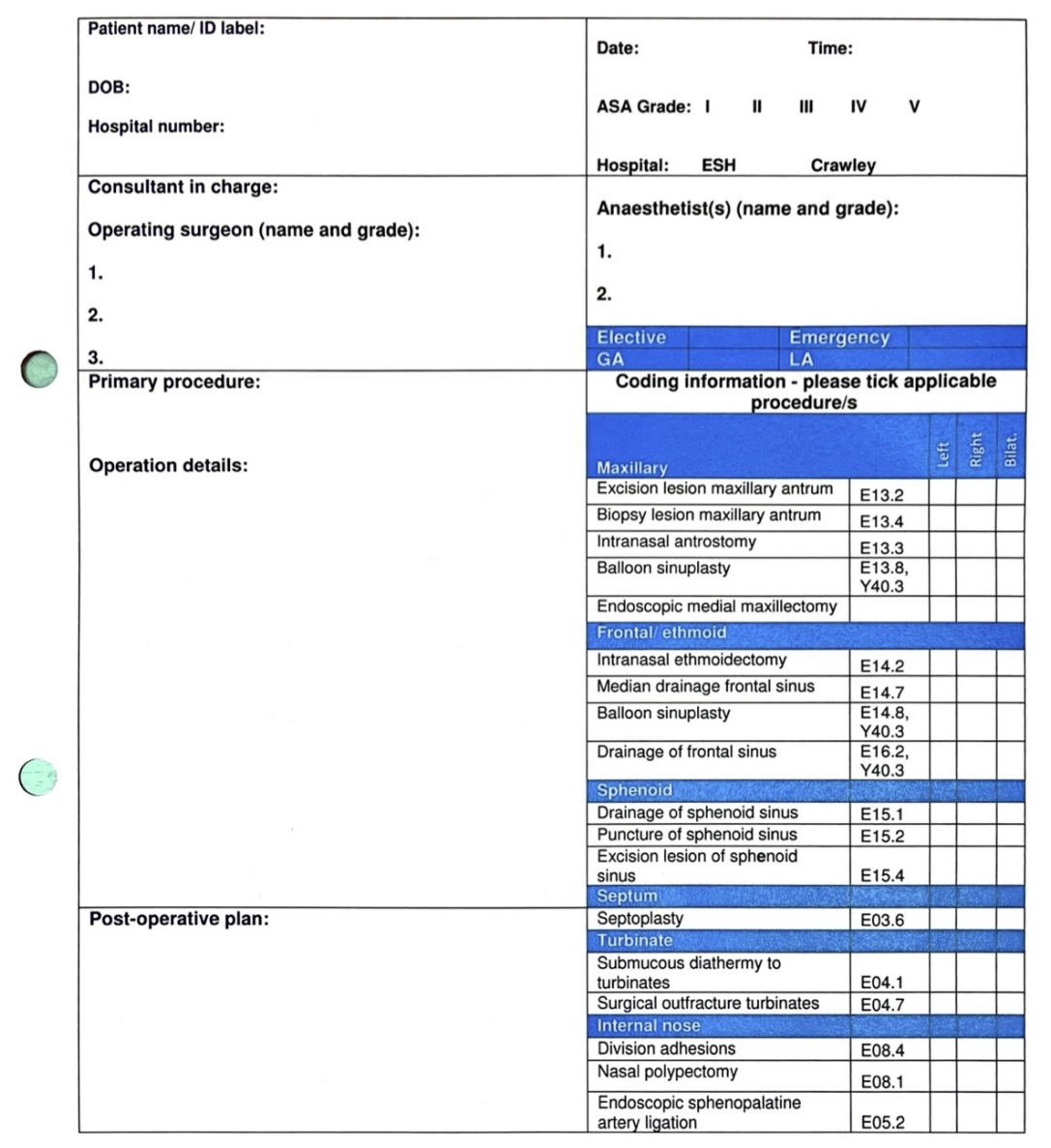

Putting people first

Delivering excellent, accessible healthcare

An Associated University Hospital of Brighton and Sussex Medical School

MR6345 